games, swim, and run with the best. I have, however, to admit of one or two exceptions. Owing to the very general tendency to low blood sugar just before the midday dinner, strenuous exercise at that time must be forbidden, as also is cycling in traffic at all times when hypoglycaemia is possible, since loss of balance is often an early symptom. It is important to minimize the psychological effect on children of knowing that they have diabetes. By showing them that when they take the diet and insulin they have made themselves normal, and, just as important, by persuading the rest of the family to treat them as if they were normal, much good can be done, and in most cases a sense of inferiority does not develop.

\section{Hypoglycaemia}

Hypoglycaemic symptoms vary to an even greater degree in children than in adults. As is well known, the blood sugar level can fall much lower in children without producing symptoms than in adults, the usual level at which symptoms are shown being about 0.05 gram per $100 \mathrm{c} . \mathrm{cm}$., though this is subject to much variation from case to case and from time to time in the same patient. Occasionally symptoms are shown at levels higher than normal-in one child at as high a level as 0.16 gram per 100 c.cm.

In addition to the usual hunger, shakiness, and hot or cold sweats, with subsequent drowsiness and coma, children often show fretfulness, tearfulness, or bad temper as early signs. Abdominal symptoms-colicky pain, nausea, vomiting, or diarrhoea-are not infrequently noted. Sometimes there are no warning symptoms and the child abruptly becomes unconscious; an observer, however, can usually see a change in the child's expression, together with pallor of the face several minutes before. The treatment is, of course, to give sugar by mouth ; it is rare for more to have to be done. However, it is not wise to. leave the child too long in the hypoglycaemic state. In one case in which the duration of hypoglycaemia was about twelve hours a further forty-eight hours elapsed before consciousness was regained, and normal mentality did not return until nearly six months had passed. A dose of $0.5 \mathrm{c.cm}$. of 1 in 1,000 adrenaline, or 10 units of pituitrin (pitressin) may have to be given, and occasionally intravenous glucose.

\section{Some Complications}

Some diabetic children suffer from periodic attacks of nausea and vomiting, with or without abdominal pain. These attacks are sometimes the outcome of hypoglycaemia. Hypoglycaemia has been shown to cause an outpouring of adrenaline, and I visualize these children as being unduly responsive to the effects of adrenaline on the alimentary tract. In some, the effect passes off with no untoward symptoms ; in others, a ketosis occurs, due both to the adrenaline itself and to the fasting entailed by the symptoms. This may bring about a typical attack of diabetic coma within as short a time as eighteen hours. Sometimes the attacks are due to excitement, or to a long journey, or to a focus of infection, thereby showing a close kinship to the periodic vomiting or bilious attacks of other children.

The condition is controlled easily in a few hours if treated at once, preferably at home. The treatment consists in giving sugar by mouth (lemonade is the easiest form) and sufficient insulin to keep the urine nearly sugar-free. The first solid meal should be easily digestible, and need not conform to the diet. As soon as the appetite returns, the normal diet is resumed. Sodium bicarbonate alone or with infusion of gentian is helpful in controlling the nausea, and will sometimes ward off an attack.

A similar set of symptoms may be associated with the onset of any acute infection, although more commonly they are absent. In either case, if a febrile condition is present extra insulin is required, and, since the diet is usually not tolerated, glucose or cane-sugar drinks must be given, together with a suitable invalid diet, until the child's condition improves. The dose of insulin is controlled by the urine tests. In general, the safest way to deal with a diabetic emergency is to administer plenty of sugar and fluids. This can do no harm so long as sufficient insulin is given to prevent the urine from showing a positive ferric chloride test. In the absence of laboratory control it is wisest to allow the urine to contain some sugar until a definite diet can be resumed. Less severe infections, such as the common cold, while not interfering with the diet, usually cause a disturbance of the carbohydrate metabolism. Extra insulin should be prescribed to correct this. Usually an increase of from 2 to 4 units will be enough to bring the urine sugar to the accustomed level. Shocks and accidents also often have a bad effect on the sugar metabolism. I have known a sudden shock in a child to precipitate a coma within twenty-four hours. If it is remembered that this may happen, it is relatively easy to control the disturbance with extra insulin and with appropriate sedative measures.

The author wishes to thank the members of the staff of the Hospital for Sick Children for permission to treat the children admitted under their care.

\title{
GLIOMA RETINAE TREATED BY RADON SEEDS
}

\author{
BY
}

H. B. STALLARD, M.A., M.D.; F.R.C.S.

ASSISTANT SURGEON, MOORFIELDS EYE HOSPITAL ; CHIEF ASSISTANT, OPHTHALMIC DEPARTMENT, ST. BARTHOLOMEW'S HOSPITAL

\section{(With Special Plate)}

Foster Moore's work at St. Bartholomew's Hospital on radiotherapy for malignant intraocular neoplasms has proved that glioma retinae is radiosensitive, and that in a number of cases this neoplasm has been completely destroyed by the application of radon seeds. An opportunity arose for studying the effects of a 3.5 millicurie seed inserted through the sclera into an eye which contained an extensive mass of glioma, and leaving this in situ for ten days before removal of the eye.

Histological investigations showed that the effective range of the radon in this case was 3 to $4 \mathrm{~mm}$. in all directions around the site of the seed. Within this zone necrobiosis, chromatolysis, granulation and vacuolation, and typical changes in irradiated cells were obvious. Outside the $4 \mathrm{~mm}$. radius the cells of the neoplasm appeared to be damaged in some places, but the histological changes were not so well marked or so uniform as within the 3 to $4 \mathrm{~mm}$. radius from the radon seed.

Other observers have given the area of destruction from radon seeds as follows.

Dose

$0.7 \mathrm{mc}$.

$2 \mathrm{mc}$.

Whatever the strength, $8 \mathrm{~mm}$. is the maximum area of necrosis.

In Foster Moore's clinic at St. Bartholomew's Hospital it is at present the practice to use radon seeds of 1 or 2 millicuries in strength, screened by platinum $0.5 \mathrm{~mm}$. thick, with a thin groove filed around the centre of the platinum envelope for the purpose of retaining a 000 black silk suture. This silk thread is tied securely in the groove, 
and to each of its free ends is threaded a half-circle arterial needle. The seeds are sewn to the exposed sclera at the desired site. From one to four seeds have been used at a time. For details of technique, post-operative complications, and results in these cases the reader is referred to the papers mentioned in the bibliography below.

\section{Case Report}

A male child, aged 2 years and 7 months, was referred to Mr. Foster Moore at St. Bartholomew's Hospital by Mr. Holmes of Liverpool on August 16th, 1934. In the absence of the former on holiday I had the opportunity of examining and treating the child. In July, 1934, the left eye had been excised for glioma retinae, and at this time the right eye was found to contain a white mass arising from the retina in the lower nasal quadrant, which was diagnosed clinically as glioma retinae. The parents refused to permit excision of this remaining eye.

The family history contained certain features of unusual interest. The father of the child had had his left eye removed for glioma retinae at the age of $\mathbf{1 4}$ months, and this diagnosis had been confirmed by pathological examination. The right eye was similarly affected, and his parents refused to allow it to be excised. At the age of 4 years he developed a very severe attack of scarlet fever, and during this attack the right eye gradually "cleared." In 1934 he was 34 years old, well, and employed as a secretary at a mental hospital. In the fundus oculi near the equator and along the course of the upper and lower nasal branches of the central retinal vessels there are now two irregularly shaped pale areas where the retina is atrophic, and there is some pigmentary disturbance. The retinal vessels traversing these areas are slightly darker in colour than normal. There is no evidence of any

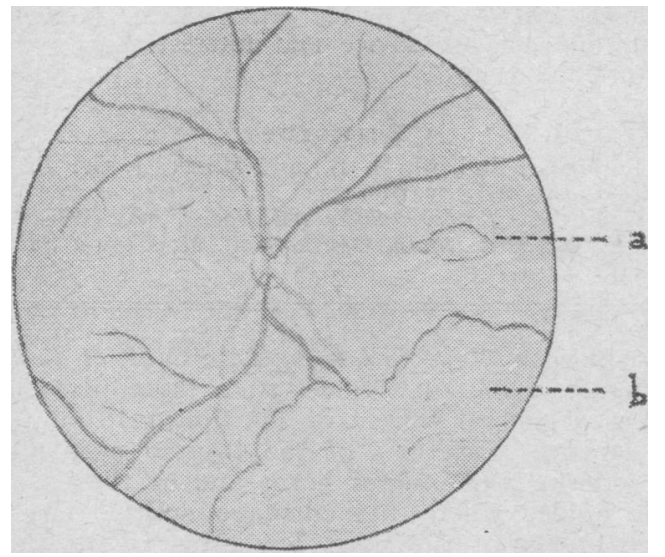

FIG. 3.-Fundus drawing. Right eye. $a$, Small island of glioma retinae in the 3 o'clock meridian just posterior to the equator. $b$, Large mass of glioma retinae occupying the lower nasal quadrant and projecting forwards into the vitreous.

intraocular neoplasm, and no defect in the visual acuity. He was seen by several ophthalmic surgeons of good repute, who had no doubts about the clinical diagnosis. (I am grateful to Messrs. Holmes and Stevenson of Liverpool for notes about this case and for permission to publish these facts.)

The second child of this man's family, the younger brother of the case here described, was noted to have bilateral glioma retinae soon after birth; both eyes were so full of the growth that little normal retina remained in either eye. On account of the parent's refusal to permit excision of the eyes, and because of the father's spontaneous cure during an attack of scarlet fever, this child was given some injections of scarlet fever serum from the age of 4 to 8 months, but he proved to be immune to this disease and so this treatment was abandoned. Injections of globulin were tried between the eighth and sixteenth months of life, twice a week at first and then once a week until there was no reaction. I am indebted to Dr. Watkins for details about this treatment. This child at the time of writing this paper was aged 2 years 4 months; he was putting on weight, and was intelligent. The neoplasm perforated the left eye posteriorly at the age of 14 months, and the right eye is full of it. $\mathrm{He}$ is quite blind, and lives in a Sunshine Home for Blind Children.
The ophthalmoscopic appearances in the remaining eye of the child, about whom this paper is concerned, are shown diagrammatically in Fig. 3.

The remainder of the fundus was normal, and the optic disk, media, and other intraocular structures were within physiological limits. The pupil was active, and the eye moved normally in all directions. There was no clinical suggestion of extraocular extension of the neoplasm.

\section{PROCEDURE ADOPTED}

It was decided to apply four 2-millicurie radon seeds to the sclera over the site of the neoplasm between the 3 o'clock and 6 o'clock meridians just behind the equator and at 7 to $8 \mathrm{~mm}$. intervals on the scleral. This was effected on August 21st, 1934, under a general anaesthetic.

The conjunctiva was incised $6 \mathrm{~mm}$. posterior to the limbus from 2.30 to 6.30 o'clock, undermined posteriorly, and retracted. The internal and inferior recti muscles were identified, and the former was divided $1 \mathrm{~mm}$. from its insertion

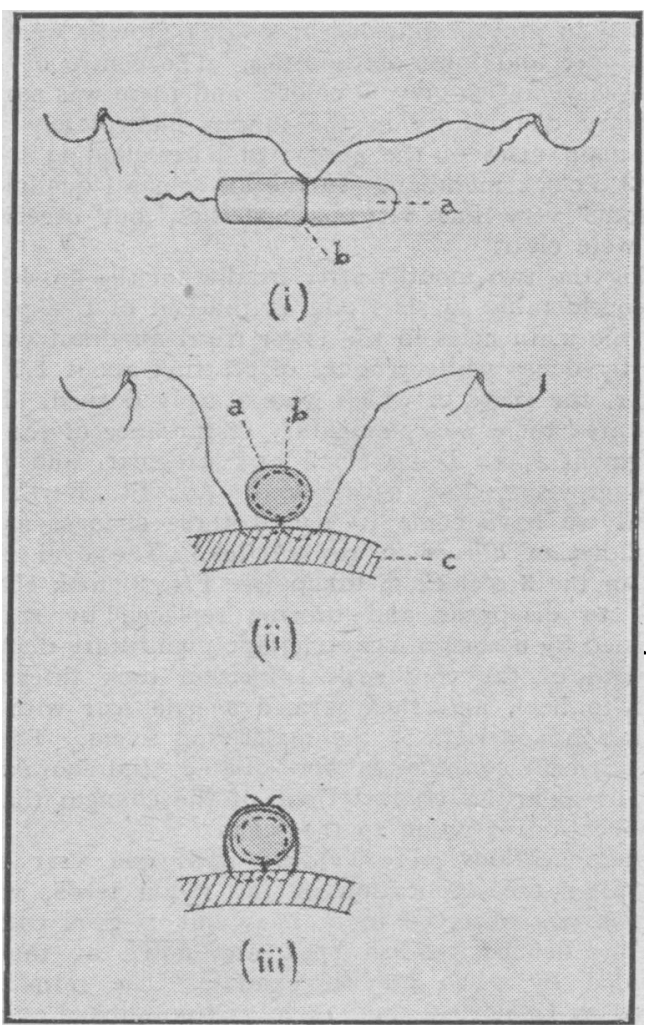

FIG. 4.-Diagrams drawn to show the method of suturing a radon seed to the sclera. (i) $a$, Radon seed. $b$, Fine black silk thread tied in a groove filed through the platinum envelope circumferentially about its centre. Arterial needles are attached to the free ends. (ii) The interrupted line represents the silk thread running in the groove around the radon seed and then passing through the superficial layers of the sclera, $c$. (iii) The ends are brought together over the seed and tied in the groove.

after passing two mattress sutures through its substance $2 \mathrm{~mm}$. behind the proposed line of section. The sclera was cleanly exposed as far back as 6 or $7 \mathrm{~mm}$. behind the equator, and absolute haemostasis was effected. The four 2-millicurie radon seeds were sutured to the sclera just posterior to the equator, with the long axis of every seed placed in the antero-posterior axis of the globe at intervals of 7 to $8 \mathrm{~mm}$. The uppermost radon seed was in line with the upper margin of the internal rectus muscle, and the lowermost in line with the temporal margin of the inferior rectus; the other two were placed in the same sagittal plane at equidistant intervals in the lower nasal quadrant (Figs. 1 and $\mathbf{2}$ on Plate).

The method of suturing the radon seeds to the sclera is shown in Fig. 4.

There was no evidence of extraocular extension of the neoplasm. The internal rectus muscle was then sutured to its divided tendon with mattress sutures, which passed forwards through the conjunctiva and were tied: The conjunctiva vas brought forward to cover the seeds, and the edges of the 
incision closed with several interrupted sutures of fine silk. Gutt. atropine sulphate 1 per cent. was instilled and the eye covered with a pad and bandaged. The elbows were encased in corrugated cardboard, and syr. chloral $3 \mathrm{j}$ with port wine $3 \mathrm{j}$ was administered four-hourly for forty-eight hours.

\section{POST-OPERATIVE SEQUELS}

The day after the operation the right lower lid was slightly oedematous, and the conjunctiva was injected over the site of the incision. The media were clear. On the third day (August 24th) the main mass of glioma appeared more densely white and opaque and was less flocculent; on the seventh day its upper extremity became broken up.

On the tenth day (August 31st) the radon seeds were removed. There was no evidence of scleral necrosis or any sign of extraocular extension of the neoplasm. The cut edges of the internal rectus muscle and the conjunctival incision were unhealed and covered with yellowish-white lymph. Conjunctivitis was evident. Fourteen days after the operation (September 4th) the neoplasm was flatter; dense white flecks embedded in greyish translucent-looking growth were present at its upper and lower extremities. The centre of the neoplasm was yellowish-grey in colour, and there was some slight pigmentary disturbance in the fundus immediately posterior to the main mass of the glioma and surrounding the island in the 3 o'clock meridian. In the lower nasal quadrant there were some very fine vitreous opacities, but otherwise the media were clear.

During the two months after irradiation the growth diminished considerably in size, and at the end of this period the site of the main mass in the lower nasal quadrant was represented by dense white p'aques of scarring about $1.5 \mathrm{~mm}$. in diameter, the edges of which were sharply defined. Posterior to this area there was pigmentary disturbance of the fundus, the pigment spots being small and irregular, and peppered over a greyish-yellow background. (At St. Bartholomew's Hospital we have come to regard these changes as typical post-irradiation effects in the fundus.) The oval island of glioma in the 3 o'clock meridian (see Fig. 3) took about four months to disappear and become replaced by scar tissue surrounded by a zone of characteristic pigmentary disturbance. The edges of the conjunctival incision took three to four months to heal, and then formed a pale scar with hyaline degeneration adherent to the underlying sclera. The vessels in the adjacent conjunctiva were dilated, and showed certain calibre irregularities characteristic of the changes that follow the application of radon to the sclera.

No complications were evident until one year and one month after the application of the radon seeds, when the optic disk was observed to be hazy but of good colour, and a sharply defined reddish spot was noted at the macula surrounded by some greyish exudate. The retinal vessels appeared to be normal, and there was no clinical evidence of recurrence. The changes mentioned above were still present on December 13th, 1935. On March 16th, 1936, a few streaklike retinal haemorrhages were seen below the macula, and a small amount of crimson blood mixed with vitreous was noted near the ora serrata about the 5 o'clock meridian over the scarred site of the main mass of the neoplasm. Some of the exudates around the macula had cleared and the edges of the optic disk were less hazy, its colour remaining within physiological limits.

For two years after treatment by radon seeds there has been no clinical evidence- of any glioma retinae elsewhere in the fundus; or a local recurrence of this neoplasm, and the child is able to see well enough to play with ordinary toys and with other children. He has been able to recognize two dogs playing in a field one-quarter of a mile away and to differentiate the size of the animals. He has remained in good health, put on weight, developed normally, and clinically has shown no sign of any intracranial extension of the glioma.

Although it is too soon to pronounce a definite opinion about the prognosis of this case, its course to date, judged by comparison with events in a few similar cases treated by radon, affords a reasonable basis for hope. Remote complications such as irradiation cataract have occurred in some cases up to eight years after irradiation near the eyes, but in these the lens may be removed and visual acuity restored provided that the retina and optic nerve are healthy. It is probable that such complications as irradiation cataract, iritis, corneal ulceration and necrosis, panophthalmitis, severe retinitis, and optic atrophy are caused by larger doses of radium or radon than we have thought fit to use in recent years.

In the case reported there was a mild degree of oedema affecting the optic disk, macula, and the papillo-macular bundle accompanied by some spots of exudate, and later a few fine retinal haemorrhages occurring one year and one month after radiotherapy, A similar complication has been noted in one of Foster Moore's glioma retinae cases, and in two cases of angiomatosis retinae treated by radon seeds. In the former, papilloedema and retinal haemorrhages were noted three months after a third application of radon by suturing four 3-millicurie radon seeds to the sclera over the site of the neoplasm in the upper nasal quadrant near the equator. (See British Journal of Ophthalmology, Monograph Supplement, "Radiant Energy ..." p. 32.) Exudates arranged around the macula in a circinate manner appeared between the third and sixth months after irradiation. The haemorrhages cleared in the course of seven months, but the exudates took two and a half years to disappear, their site being represented by some pigmentary disturbance. In one of the angiomatosis retinae cases, exudate at the macula appeared ten months after the scleral application of three 5.1-millicurie radon seeds to the upper nasal quadrant $10 \mathrm{~mm}$. from the limbus in the $2.30 \mathrm{o}$ 'clock meridian of the right eye. These exudates cleared during the next three months.

I thank Mr. Foster Moore for permission to publish this case, which was treated in his wards at St. Bartholomew's Hospital, and for his advice and interest during the postoperative " follow-up."

BibLIOGRAPHY

Moore R. Foster Stallard, H. B, and Milner, J. G.: Brit. Journ. Ophthalmol., 1931, xv, 673

More, R. Foster. Trans, Ophthalmol. Scc. U. K., 1935, lv, 3. Stallard, H. B.: Brit. Journ. Ophthalmol., Monograph Supplement, Radiant Energy.

A. Laskiewicz (Rev. de Laryngol., d'Otol. et de Rhinol., July-August, 1936, p. 749) describes three personal cases and gives a general survey of the literature on fractures of the larynx. Fractures of the thyroid cartilage occur in the superior and inferior horns and at the base of the anterior angle, where the cartilage splits. The cricoid cartilage breaks at the sides, less often in the thickened posterior portion when the fracture is accompanied by a luxation of the crico-arytenoid articulation. The hyoid bone is well protected behind the mandible and is seldom involved, but it has been known to become fractured by violent contraction of the pharyngeal constrictor muscles in vomiting. At the time of the injury' there is always loss of consciousness through reflex inhibition of the vagus (laryngeal shock); later one finds loss of voice, pain, haemoptysis, cyanosis. An interesting sign is sub cutaneous emphysema, occurring more particularly with injuries of the cricoid cartilage when there has been a break in the mucous membrane. In the complicated fractures with haemorrhage and emphysema the lower air passages may become gradually occluded, and a tracheotomy cannot save the patient. Later complications are pneumonia and mediastinitis, also septicaemia from continued suppuration of separated fragments of cartilage. Marschik estimates that the mortality of fractures of the larynx is as high as 37 per cent. As regards treatment, an emergency tracheotomy is nearly always necessary. In the more complicated cases one may have to perform a laryngofissure in addition. This allows one to remove loose fragments of cartilage. Pieces of cartilage still attached by perichondrium are pushed into place and held there by packing. According to the author such fragments must never be fixed by sutures, as this causes obstinate fistulae in the neck. 
H. B. STALLARD : GLIOMA RETINAF: TREATED BY RADON SEEDS

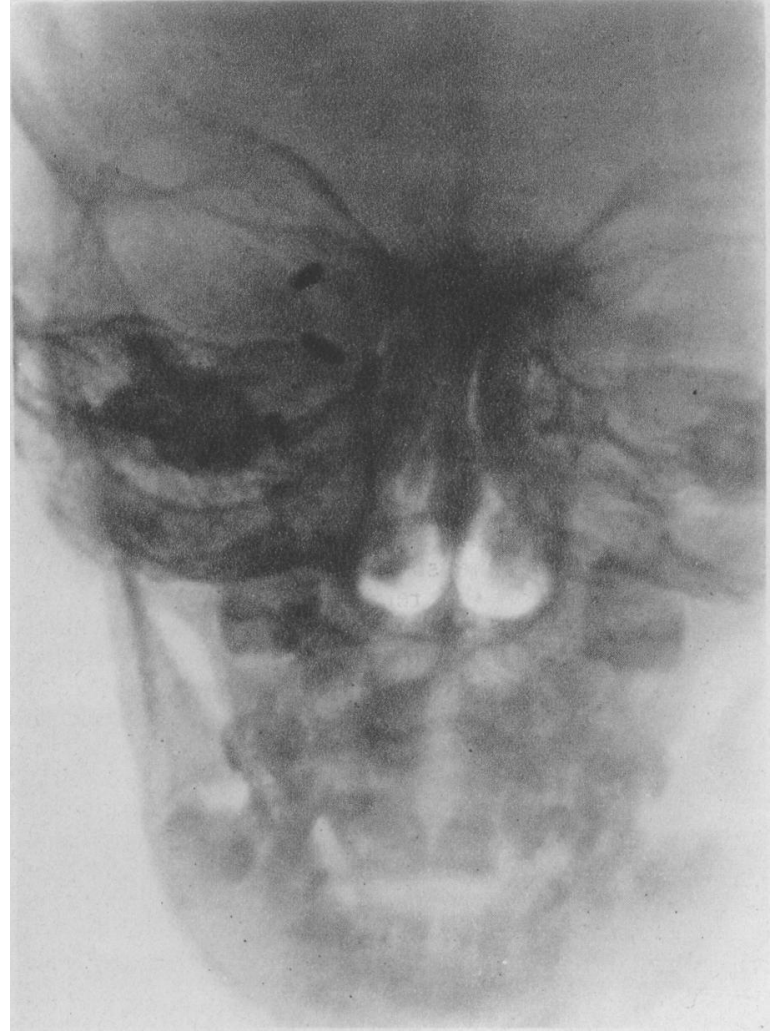
Fia. 1.-Antero-posterior view. Four radon seeds in situ

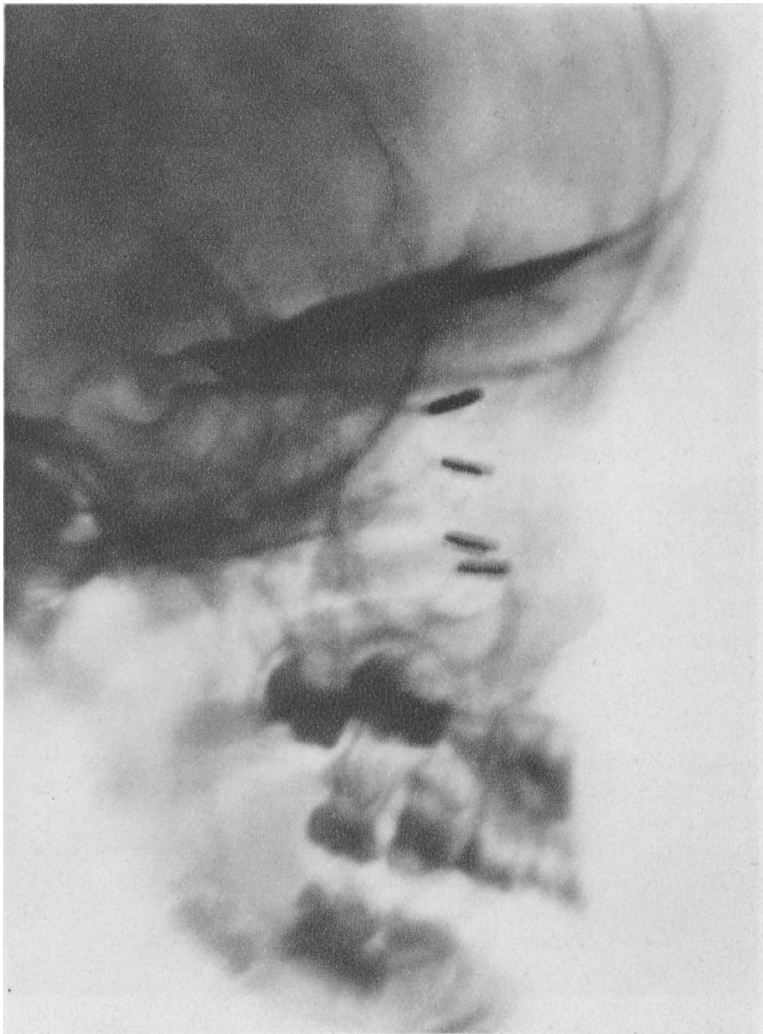

Fra. 2.-Lateral view. Head moved.

A. G. WA'TKINS : SPONTANEOUS SURGICAL EMPHYSEMA IN CHILDREN

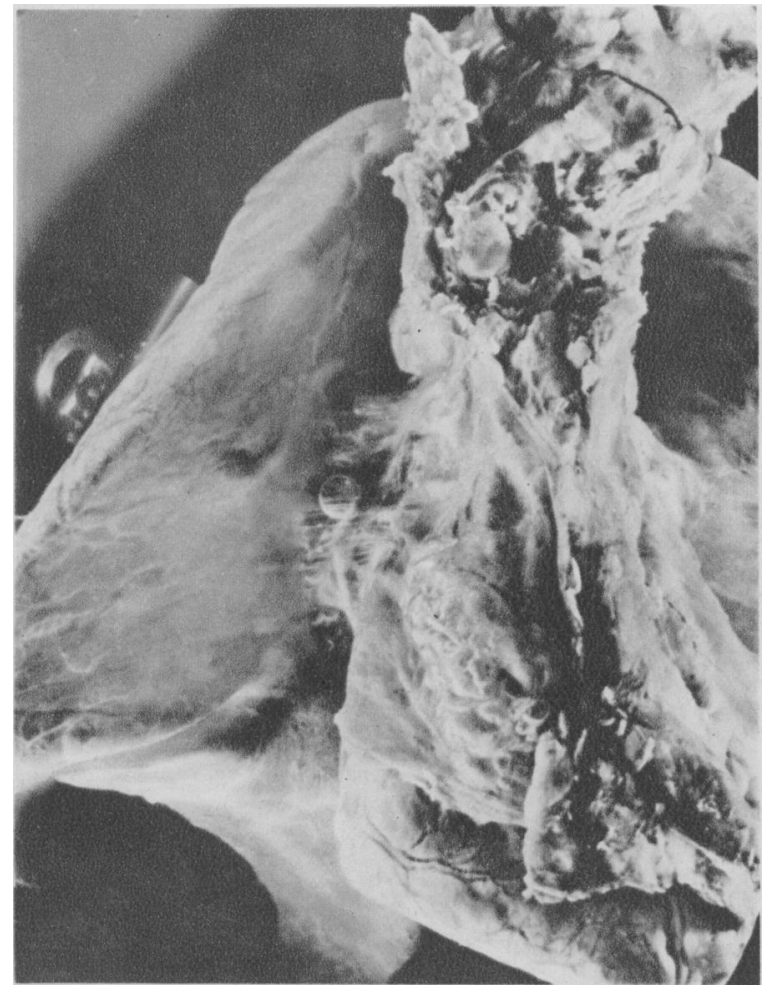

Fia. 1.-Case III, showing bullae in mediastinal tissue and medial aspect of lung.

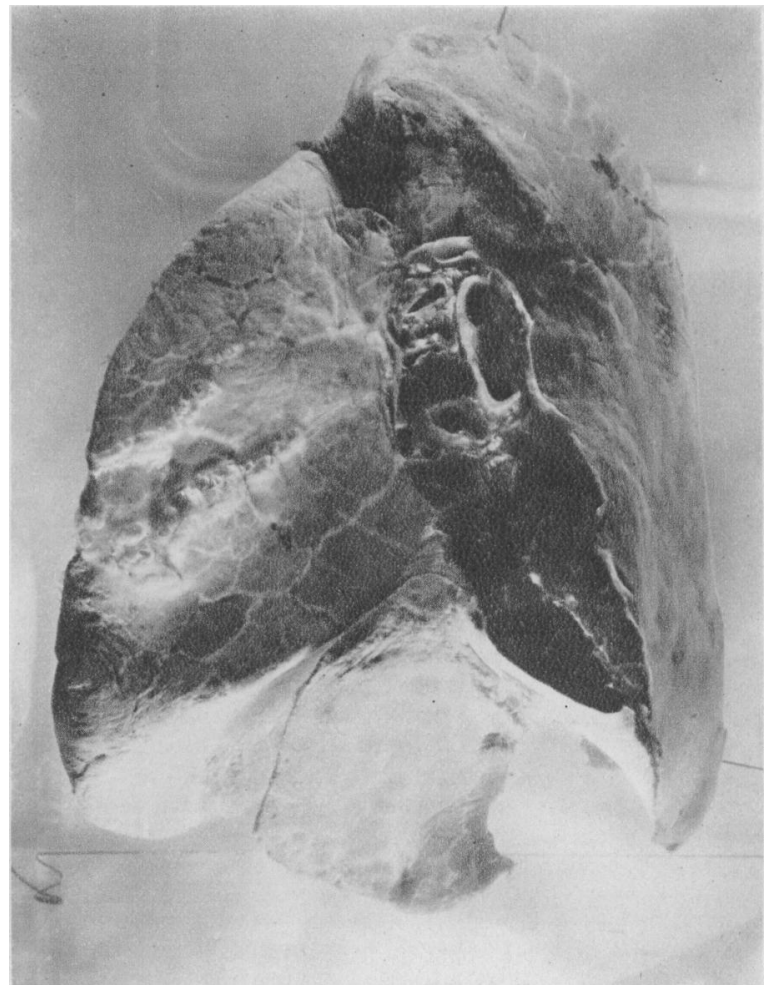

Fia. 2.-C'ase IV, showing interstitial emphysema. 